\title{
A Review: Detecting Alterations of Brain Connectivity in Schizophrenia based on Structural MRI
}

\author{
Chunlan Yang ${ }^{1^{*}}$, Wan $\mathrm{Li}^{1}$, Wu Weiwei ${ }^{1}$, Shuicai $\mathrm{Wu}^{{ }^{1 *}}$ and Wangsheng $\mathrm{Lu}^{2}$ \\ ${ }^{1}$ College of Life Science and Bioengineering, Beijing University of Technology, Beijing, China \\ ${ }^{2}$ Center of Neurosurgery, PLA NAVY General Hospital, Beijing, China
}

"Corresponding author: Chunlan Yang or Shuicai Wu, College of Life Science and Bioengineering, Beijing University of Technology, Beijing, China, 100022, Tel: 86-10-67391610; Fax: 86-10-67391610; E-mail: clyang@bjut.edu.cn; shuicaiwu@bjut.edu.cn

Received date: September 2, 2014, Accepted Date: September 23, 2014, Published Date: September 30, 2014

Copyright: ( 2014 Yang C et al. This is an open-access article distributed under the terms of the Creative Commons Attribution License, which permits unrestricted use, distribution, and reproduction in any medium, provided the original author and source are credited.

\begin{abstract}
Schizophrenia is a common psychiatric disease with brain connectivity changed. Nowadays brain network has become an effective tool to detect the abnormal brain connectivity in patients. Compared with studies focused on functional and white matter connectivity, the research of grey matter connectivity is relatively less and should be paid more attention. The primary approach of grey matter connectivity analysis is based on brain network constructed from structural MRI (sMRI). There are several morphometric features such as cortical thickness, volume, and curvature etc. Which could be used for SMRI brain network construction? This review briefly introduced sMRI brain network construction and analysis, presenting the most frequently used morphometric features with their effectiveness in schizophrenia, highlighting the application of sMRI brain network in schizophrenia, and finally estimated its potential value.
\end{abstract}

Keywords: Schizophrenia; Structural MRI; Brain network; Morphometric features

\section{Introduction}

Schizophrenia is a complex condition with a wide range of clinical signs [1]. Generally it was defined as a chronic and debilitating mental disorder, which usually has an onset in adolescence or early adulthood [2]. It involves abnormal emotional responses and difficulty with social interactions [3]. In addition, patients with schizophrenia exhibit impairments in both basic sensory processing and higher cognitive functions, such as language, reasoning and planning [4]. Nowadays, there are some brain image systems helping to diagnose of schizophrenia, like structural MRI [5], diffusion MRI [6], functional MRI [7], PET [8], MEG [9] and SPECT [10] etc. However, the etiology and pathophysiology of schizophrenia remain unknown [2], while indeed, schizophrenia has come to be regarded more and more as a disease of disconnectivity [11]. Therefore, it makes brain network play an extremely important role in detection of altered brain connectivity in schizophrenia.

For different diagnosis purpose, various brain networks have been developed. To date, there are mainly three forms of network: functional network derived from BOLD-MRI (Blood- Oxygen- Level Dependent (fMRI) [12-14]; structural network derived from diffusion MRI (DTI) focused on white matter [15-17]; and structural network derived from structural MRI (sMRI) focused on gray matter [18-20]. Compared with sMRI-based network, DTI-based and fMRI-based networks are easier to build because it is necessary to extract one or more reasonable morphometric features for construction of sMRIbased network. For this reason, sMRI-based network is relatively less popular used although sMRI has the merit of higher resolution. But with the development of neuroimaging technique, it will be widely used and attach the equal importance with DTI-based and fMRI-based networks.
In this review, we concentrated on application of sMRI-based network in schizophrenia. First, the construction of sMRI-based network was briefly outlined. Second, the frequently used morphometric features were elucidated. Third, analysis of brain connectivity alteration in schizophrenia using sMRI-based network in recently researches were presented. Finally, the problems and future development of sMRI-based brain network were pointed out.

\section{Structural MRI Network}

Alexander et al. [11] revealed that networks construction and analyses generally used three steps: seed analysis, principal component analysis (PCA)and graph analysis. These procedures are not exclusive to sMRI network and more details were represented in the following sections.

\section{Seed Analysis}

To construct the network, firstly we need to determine the 'nodes' of the network according to brain parcellation. Automated anatomical labeling (AAL) [21] is the most commonly used parcellation template.Besides, there are also Harvard Oxford (H-O) [22], EickhoffZilles (E-Z) [23], Talariach-Tournoux (T-T) [24] and CC200 (or CC400) [25] in use. After parcellation, each node needs to be defined as at least one morphometric feature. Therefore, the morphology in the regions could be compared with each other and then a whole-brain map of structural co-variance could be generated [11].

\section{Principal Component Analysis}

PCA uses an orthogonal transformation to convert a set of observations of possibly correlated variables into a set of values of linearly uncorrelated variables called principal components [26]. This theory was invented in 1901 by Karl Pearson [27]. In brain network construction, PCA reduces the inter-regional co-variance 
Page 2 of 5

across people to a small number of factors which are easier to visualize and interpret [11].

\section{Graphic Theory}

Graphic theory provides a powerful tool to quantitatively describe the topological organization of brain network connectivity $[28,29]$. Clustering coefficient [30], shortest path length [31], small-worldness [32], degree [33], nodal efficiency [34] and betweenness centrality [35] are the most frequently used parameters to describe the topological properties of brain network. Moreover, the abnormal regions could be identified using brain network.

\section{Morphometric Features}

Various forms of brain network have been established based on the corresponding brain features. For DTI-based brain network, the tensor of water molecules in fiber bundles of white matter is the feature which represents how seed regions connect to each other. And in fMRI-based brain network, the correlation between BOLD signals is the feature to present whether the seed regions have the connectivity. While for sMRI-based brain network, the feature is extracted from grey matter based on morphometry.

Levitt et al. proposed that schizophrenia is believed to be a disorder which many regions of the brain affected [36]. There have been large amount of imaging studies assessing brain morphometry to detect abnormalities in multiple regions in this devastating disorder. Therefore, morphometry, and specifically, morphometric feature is the basis of the sMRI-based brain network construction and analysis. There are diverse morphometric features because brain morphometry can be measured through multiple ways, which turns out to be an advantage of sMRI that can tell us more than one aspect of brain condition. We will elaborate the common morphometric features with references as following.

\section{Cortical Thickness}

Cortical thickness was estimated as the shortest distance between the gray and white matter border and the pial surface at numerous points across the entire cortical mantle [37]. Its variation across the human brain follows small-world principles [38]. Kuperberg et al. have found significant thinning in distributed areas of the cortex, most prominently in frontal and temporal region sinchronic schizophrenia patients [39]. Nesvåg et al. found thinner cortex located in prefrontal and temporal regions of both hemispheres in schizophrenia patients, while parietal and occipital regions were relatively spared [37]. But Wieg and et al. did not detect cortical thinning averaged across the entire prefrontal lobe in first episode schizophrenia patients [40].

\section{Volume}

Brain size is measured by volume via MRI scans. Van Haren et al. suggested that brain maturation occurring in the third and fourth decade of life is abnormal in schizophrenia, based on the longitudinal study that different age-related trajectories of brain tissue loss are present in patients compared to healthy subjects [41]. Scheewe et al. found significantly smaller baseline cerebral (grey) matter, and larger third ventricle volumes, and thinner cortex in most areas of the brain in patients with schizophrenia [42]. Abbs et al. [43] found that in schizophrenia, anterior cingulate gyrus (ACG) volume was reduced in females, but not in men, relative to controls.

\section{Curvature}

Curvature of the brain surface provides an effective method for assessing the character of convolutions on the brain's surface, thereby serving as an index of normal versus abnormal brain development [44]. Ronan et al. found that millimeter-scale intrinsic curvature measures were more robust and consistent in identifying reduced gyrification in patients with schizophrenia [45].

\section{Complexity}

Cortical complexity is a measurement which could quantify the spatial frequency of gyrification and fissuration of the brain surface [46]. Wiegand et al. found prefrontal cortical complexity was not significantly different among the groups including both subtypes of patients and healthy controls [47]. However, the schizophrenia patients differed significantly from the healthy subjects in asymmetry, showing less left-greater-than-right asymmetry in cortical complexity than the controlled subjects.

\section{Density}

After the individual images were segmented and registered, each voxel then achieved a measure of the probability, according to which it belongs to a certain tissue class. For gray matter, this quantitative measurement is usually represented as gray matter density (GMD) or gray matter concentration (GMC), or gray matter probability (GMP) [48]. Stegmayer et al. had the main finding which suggests severe emotional disturbance in schizophrenia be particularly associated with reduced GMD in a large cluster including the ventral striatum [49].

\section{Cortical folding}

The degree of folding relative to brain size remains relatively stable from early childhood [50,51], and is thus a suitable subject for investigation of brain disorder. Abnormalities like schizophrenia might denote abnormal cortical folding development, which can now be investigated using gyrification measures [52]. Nesvåg et al. [53] found the reduced degree of folding in large regions of the cerebral cortex across two independent samples indicates that reduced gyrification is an inherent feature of the brain pathology in schizophrenia.

\section{sMRI-based Brain Network Application in Schizophrenia}

With the development of morphometric feature studies, brain network helps us get more information about the whole brain connection. Besides, discoveries of structural MRI-based network in schizophrenia can complement studies which show disrupted white matter tracts [3] and functional connectivity [54] between brain regions in the disease [11]. Then, we will present the results of recent researches of sMRI-based network applied in the schizophrenia.

Salgado et al. compared grey matter volumes using voxel-based morphometry (VBM) and discovered volume reductions in medial cortical regions which overlapped with the same parts of the functional network in the patients $[19,55]$. Bassett et al. [56] constructed the anatomical networks derived from analysis of inter-regional co variation of gray matter volume. They proposed that the topological differences between divisions of normal cortex may represent the different growth processes. What's more, neuro developmental abnormalities in schizophrenia specifically impact multimodal cortical organization. Shi et al. [57] indicated that the 
brain structural associations of the high-risk neonates tended to have globally lower efficiency, longer connection distance, and less number of hub nodes and edges with relatively higher betweenness by the morphological network analysis. Zhang et al. constructed the brain networks by thresholding cortical thickness correlation matrices and they found both characteristic path length and clustering coefficient increased in the structural cortical networks of patients [18]. Moreover, in two years later, Zhang et al. also found that less distributed cortical regions were identified in the thalamo-cortical network in patients with schizophrenia, but vertex-wise comparison revealed decreased thalamo-cortical connectivity in bilateral inferior frontal gyrus, the left superior temporal gyrus and the right parietooccipital region, by constructing a thalamo-cortical network to assess the correlation between the thalamic volume and cortical thickness at each vertex on the cortical surface [58]. Rüsch et al. did a research on regional gray matter volumes [59] and analyzed by VBM within SPM5 (statistical parametric mapping) [60], the results show that patients with schizophrenia have reduced gray matter volume in dorso lateral prefrontal and anterior cingulate. Jagannathan et al. found gray matter deficited in patients with schizophrenia consistently with previous reports, including frontal and temporal lobes and thalamus [61]. Bagary et al. found that smooth pursuit abnormalities were associated with reduced magnetization transfer ratio in several regions, predominantly in the right prefrontal cortex [62]. Collin et al. discovered schizophrenia patients showing both decreased (e.g. between left frontal and bilateral subcortical, $\mathrm{p} \leq 0.005$ ) and increased (e.g. between left temporal and bilateral subcortical, $\mathrm{p} \leq 0.001$ ) coupling between lobar grey matter volumes [63]. Glahn et al. found patients had reduced gray matter density in a distributed network of regions (including bilateral insular cortex, anterior cingulate, left parahi ppocampal gyrus, left middle frontal gyrus, postcentral gyrus, and thalamus) and increased gray matter density in striatal regions [64].

\section{Conclusions}

Brain network construction and analyses is becoming an essential tool recently, to help diagnosing the nerve system disorder and orientating the foci, such as schizophrenia which might not has apparent brain damages presented on MRI or other images. For sMRIbased brain network, according to the recent researches, the detected localization of foci, like frontal lobes, thalamus, hippocampus and temporal lobes, is generally same with the result by fMRI-based network. Altered morphometric features such as grey matter reduction and density change were discovered, and the network topology analysis of patients showed lower globally efficiency, longer connection distance, less number of hub nodes and edges with relatively higher betweenness, increased characteristic path length and clustering coefficient.

Compared with the other two forms of brain network, the development of sMRI-based brain network is a little bit lagged behind. On the other way, it still has so many interesting and amazing properties remained to explore. At present, sMRI-based network is constructed on inter-regional correlations estimated from a group of individual images [11], which means it's not feasible for individual. Although there are some studies proposed methods $[65,66]$ to solve this problem, they still have limitations and needs to be improved for application. Another problem is that, morphometric features used in network construction were generally focused on cortical thickness and volume, which more sensitive morphotric features able to describe the disease should be considered.

\section{Acknowledgment}

This work is partially supported by the Natural Science Foundation of China (Grant No. 81101107) and Natural Science Foundation of Beijing (Grant No. 3112005).

\section{References}

1. Briki M, Monnin J, Haffen E, Sechter D, Favrod J, et al. (2014) Metacognitive training for schizophrenia: a multicentre randomised controlled trial. Schizophr Res 157: 99-106.

2. Cookey J, Bernier D, Tibbo PG (2014) White matter changes in early phase schizophrenia and cannabis use: An update and systematic review of diffusion tensor imaging studies. Schizophrenia research 156: 137-142.

3. Ohtani T, Bouix S, Hosokawa T, Saito Y, Eckbo R, et al. (2014) Abnormalities in white matter connections between orbitofrontal cortex and anterior cingulate cortex and their associations with negative symptoms in schizophrenia: A DTI study. Schizophrenia research 153: 1-3.

4. Uhlhaas PJ, Singer W (2010) Abnormal neural oscillations and synchrony in schizophrenia. Nat Rev Neurosci 11: 100-113.

5. Mahon PB, Eldridge H, Crocker B, Notes L, Gindes H, et al. (2012) An MRI study of amygdala in schizophrenia and psychotic bipolar disorder. Schizophr Res 138: 188-191.

6. Pasternak O, Westin CF, Dahlben B, Bouix S, Kubicki M (2014) The extent of diffusion MRI markers of neuroinflammation and white matter deterioration in chronic schizophrenia. Schizophr Res .

7. Das P, Lagopoulos J, Coulston CM, Henderson AF, Malhi GS (2012) Mentalizing impairment in schizophrenia: a functional MRI study. Schizophr Res 134: 158-164.

8. Nozaki S, Kato M, Takano H, Ito H, Takahashi H, et al. (2009) Regional dopamine synthesis in patients with schizophrenia using L[beta-11C]DOPA PET. Schizophr Res 108: 78-84.

9. Koh Y, Shin KS, Kim JS, Choi JS, Kang DH, et al. (2011) An MEG study of alpha modulation in patients with schizophrenia and in subjects at high risk of developing psychosis. Schizophr Res 126: 36-42.

10. Wake R, Miyaoka T, Kawakami K, Tsuchie K, Inagaki T, Horiguchi (2010) Characteristic brain hypoperfusion by $99 \mathrm{mTc}-\mathrm{ECD}$ single photon emission computed tomography (SPECT) in patients with the firstepisode schizophrenia. European Psychiatry 25: 361-365.

11. Alexander-Bloch A, Giedd JN, Bullmore E (2013) Imaging structural covariance between human brain regions. Nat Rev Neurosci 14: 322-336.

12. Shim M, Kim DW, Lee SH, Im CH (2014) Disruptions in small-world cortical functional connectivity network during an auditory oddball paradigm task in patients with schizophrenia. Schizophrenia research 156: 197-203.

13. de Arruda GF, Fontoura Costa Ld, Schubert D, Rodrigues FA (2014) Structure and dynamics of functional networks in child-onset schizophrenia. Clin Neurophysiol 125: 1589-1595.

14. Chen YL, Tu PC, Lee YC, Chen YS, Li CT (2013) Resting-state fMRI mapping of cerebellar functional dysconnections involving multiple large-scale networks in patients with schizophrenia. Schizophrenia research 149: 26-34.

15. Leroux E, Delcroix N, Dollfus S (2014) Left fronto-temporal dysconnectivity within the language network in schizophrenia: an fMRI and DTI study. Psychiatry Res 223: 261-267.

16. Hoptman MJ, Nierenberg J, Bertisch HC, Catalano D, Ardekani BA, et al. (2008) A DTI study of white matter microstructure in individuals at high genetic risk for schizophrenia. Schizophr Res 106: 115-124.

17. Kubicki M, Park H, Westin CF, Nestor PG, Mulkern RV, et al. (2005) DTI and MTR abnormalities in schizophrenia: analysis of white matter integrity. Neuroimage 26: 1109-1118. 
18. Zhang Y, Lin L, Lin CP, Zhou Y, Chou KH, et al. (2012) Abnormal topological organization of structural brain networks in schizophrenia. Schizophr Res 141: 109-118.

19. Salgado-Pineda P, Fakra E, Delaveau P, McKenna PJ, Pomarol-Clotet E, et al. (2011) Correlated structural and functional brain abnormalities in the default mode network in schizophrenia patients. Schizophr Res 125: 101-109.

20. Martin AK, Robinson G, Reutens D, Mowry B (2014). Copy number deletion burden is associated with cognitive, structural, and resting-state network differences in patients with schizophrenia. Behavioural brain research.

21. Tzourio-Mazoyer N, Landeau B, Papathanassiou D, Crivello F, Etard O, et al. (2002) Automated anatomical labeling of activations in SPM using a macroscopic anatomical parcellation of the MNI MRI single-subject brain. Neuroimage 15: 273-289.

22. Desikan RS, Ségonne F, Fischl B, Quinn BT, Dickerson BC, et al. (2006) An automated labeling system for subdividing the human cerebral cortex on MRI scans into gyral based regions of interest. Neuroimage 31: 968-980.

23. Eickhoff SB, Stephan KE, Mohlberg H, Grefkes C, Fink GR, et al. (2005) A new SPM toolbox for combining probabilistic cytoarchitectonic maps and functional imaging data. Neuroimage 25: 1325-1335.

24. Lancaster JL, Woldorff MG, Parsons LM, Liotti M, Freitas CS, et al. (2000) Automated Talairach atlas labels for functional brain mapping. Hum Brain Mapp 10: 120-131.

25. Craddock RC, James GA, Holtzheimer PE 3rd, Hu XP, Mayberg HS (2012) A whole brain fMRI atlas generated via spatially constrained spectral clustering. Hum Brain Mapp 33: 1914-1928.

26. Wold S (1987) Principal Component Analysis. Chemometrics and Intelligent Laboratory Systems 2: 37-52.

27. Pearson K (1901) LIII. On lines and planes of closest fit to systems of points in space. The London, Edinburgh, and Dublin Philosophical Magazine and Journal of Science 2: 559-572.

28. Bullmore E, Sporns O (2009) Complex brain networks: graph theoretical analysis of structural and functional systems. Nat Rev Neurosci 10: 186-198.

29. Bassett DS, Gazzaniga MS (2011) Understanding complexity in the human brain. Trends Cogn Sci 15: 200-209.

30. Onnela JP, Saramäki J, Kertész J, Kaski K (2005) Intensity and coherence of motifs in weighted complex networks. Phys Rev E Stat Nonlin Soft Matter Phys 71: 065103.

31. Zhang Z, Liao W, Chen H, Mantini D, Ding JR, et al. (2011) Altered functional-structural coupling of large-scale brain networks in idiopathic generalized epilepsy. Brain 134: 2912-2928.

32. Watts DJ, Strogatz SH (1998) Collective dynamics of 'small-world' networks. Nature 393: 440-442.

33. Rubinov M, Sporns O (2010) Complex network measures of brain connectivity: uses and interpretations. Neuroimage 52: 1059-1069.

34. Shu N, Liu Y, Li K, Duan Y, Wang J, et al. (2011) Diffusion tensor tractography reveals disrupted topological efficiency in white matter structural networks in multiple sclerosis. Cerebral Cortex 21: 2565-2577.

35. Freeman LC (1977) A set of measures of centrality based on betweenness. Sociometry 35-41.

36. Levitt JJ, Bobrow L, Lucia D, Srinivasan P (2010) A selective review of volumetric and morphometric imaging in schizophrenia. Curr Top Behav Neurosci 4: 243-281.

37. Nesvåg R, Lawyer G, Varnäs K, Fjell AM, Walhovd KB, et al. (2008) Regional thinning of the cerebral cortex in schizophrenia: effects of diagnosis, age and antipsychotic medication. Schizophr Res 98: 16-28.

38. He Y, Chen ZJ, Evans AC (2007) Small-world anatomical networks in the human brain revealed by cortical thickness from MRI. Cereb Cortex 17: 2407-2419.

39. Kuperberg GR, Broome MR, McGuire PK, David AS, Eddy M, et al. (2003) Regionally localized thinning of the cerebral cortex in schizophrenia. Arch Gen Psychiatry 60: 878-888.
40. Wiegand LC, Warfield SK, Levitt JJ, Hirayasu Y, Salisbury DF, et al. (2004) Prefrontal cortical thickness in first-episode psychosis: a magnetic resonance imaging study. Biol Psychiatry 55: 131-140.

41. Van Haren NE, Cahn W, Hulshoff Pol HE, Kahn RS (2013) Confounders of excessive brain volume loss in schizophrenia. Neurosci Biobehav Rev 37: 2418-2423.

42. Scheewe TW, van Haren NE, Sarkisyan G, Schnack HG, Brouwer RM, et al. (2013) Exercise therapy, cardiorespiratory fitness and their effect on brain volumes: a randomised controlled trial in patients with schizophrenia and healthy controls. European Neuro psychopharmacology 23: 675-685.

43. Abbs B, Liang L, Makris N, Tsuang M, Seidman LJ, et al. (2011) Covariance modeling of MRI brain volumes in memory circuitry in schizophrenia: Sex differences are critical. Neuroimage 56: 1865-1874.

44. Pearlson GD, Marsh L (1999) Structural brain imaging in schizophrenia: a selective review. Biol Psychiatry 46: 627-649.

45. Ronan L, Voets NL, Hough M, Mackay C, Roberts N, et al. (2012). Consistency and interpretation of changes in millimeter-scale cortical intrinsic curvature across three independent datasets in schizophrenia. Neuroimage, 63: 611-621.

46. Luders E, Narr KL, Thompson PM, Rex DE, Jancke L, et al. (2004) Gender differences in cortical complexity. Nat Neurosci 7: 799-800.

47. Wiegand LC, Warfield SK, Levitt JJ, Hirayasu Y, Salisbury DF, et al. (2005) An in vivo MRI study of prefrontal cortical complexity in firstepisode psychosis. Am J Psychiatry 162: 65-70.

48. Wikipedia, Brain Morphrmetry.

49. Stegmayer K, Horn H, Federspiel A, Razavi N, Bracht T, et al. (2013) Ventral striatum gray matter density reduction in patients with schizophrenia and psychotic emotional dysregulation. Neuroimage Clin 4: 232-239.

50. Armstrong E, Schleicher A, Omran H, Curtis M, Zilles K (1995) The ontogeny of human gyrification. Cereb Cortex 5: 56-63.

51. Zilles K, Palomero-Gallagher N, Amunts K (2013) Development of cortical folding during evolution and ontogeny. Trends Neurosci 36: 275-284.

52. Cachia A, Paillère-Martinot ML, Galinowski A, Januel D, de Beaurepaire $\mathrm{R}$, et al. (2008) Cortical folding abnormalities in schizophrenia patients with resistant auditory hallucinations. See comment in PubMed Commons below Neuroimage 39: 927-935.

53. Nesvag R, Schaer M, Haukvik UK, Westlye LT, Rimol LM, et al. (2014) Reduced brain cortical folding in schizophrenia revealed in two independent samples. Schizophrenia research 152: 333-338.

54. Ma S, Calhoun VD, Phlypo R, Adal T (2014). Dynamic changes of spatial functional network connectivity in healthy individuals and schizophrenia patients using independent vector analysis. NeuroImage 90: 196-206.

55. Ridgway GR, Henley SM, Rohrer JD, Scahill RI, Warren JD, et al. (2008) Ten simple rules for reporting voxel-based morphometry studies. Neuroimage 40: 1429-1435.

56. Bassett DS, Bullmore E, Verchinski BA, Mattay VS, Weinberger DR, et al. (2008) Hierarchical organization of human cortical networks in health and schizophrenia. J Neurosci 28: 9239-9248.

57. Shi F, Yap PT, Gao W, Lin W, Gilmore JH, et al. (2012) Altered structural connectivity in neonates at genetic risk for schizophrenia: a combined study using morphological and white matter networks. Neuroimage 62: 1622-1633.

58. Zhang Y, Su TP2, Liu B3, Zhou Y4, Chou KH5, et al. (2014) Disrupted thalamo-cortical connectivity in schizophrenia: a morphometric correlation analysis. Schizophr Res 153: 129-135.

59. Rüsch N, Spoletini I, Wilke M, Bria P, Di Paola M, et al. (2007) Prefrontal-thalamic-cerebellar gray matter networks and executive functioning in schizophrenia. Schizophr Res 93: 79-89.

60. Friston KJ (2003). Statistical parametric mapping. In Neuroscience Databases (pp. 237-250). Springer US. 
Citation: Yang C, Li W, Weiwei W, Wu S, Lu W (2014) A Review: Detecting Alterations of Brain Connectivity in Schizophrenia based on Structural MRI. J Psychiatry 17: 1000159. doi:10.4172/1994-8220.1000159

Page 5 of 5

61. Jagannathan K, Calhoun VD, Gelernter J, Stevens MC, Liu J, et al. (2010) Genetic associations of brain structural networks in schizophrenia: a preliminary study. Biol Psychiatry 68: 657-666.

62. Bagary MS, Hutton SB, Symms MR, Barker GJ, Mutsatsa SH, et al. (2004). Structural neural networks subserving oculomotor function in first-episode schizophrenia. Biological psychiatry 56: 620-627.

63. Collin G, de Reus MA, Cahn W, Hulshoff Pol HE, Kahn RS, et al. (2013) Disturbed grey matter coupling in schizophrenia. Eur Neuropsychopharmacol 23: 46-54.

64. Glahn DC, Laird AR, Ellison-Wright I, Thelen SM, Robinson JL, et al. (2008). Meta-analysis of gray matter anomalies in schizophrenia: application of anatomic likelihood estimation and network analysis. Biological psychiatry 64: 774-781.

65. Batalle D, Muñoz-Moreno E, Figueras F, Bargallo N, Eixarch E, et al. (2013) Normalization of similarity-based individual brain networks from gray matter MRI and its association with neurodevelopment in infants with intrauterine growth restriction. Neuroimage 83: 901-911.

66. Tijms BM, Seriès P, Willshaw DJ, Lawrie SM (2012) Similarity-based extraction of individual networks from gray matter MRI scans. Cereb Cortex 22: 1530-1541. 